\title{
Clinical characteristics of primary hepatic angiosarcoma outcomes: a SEER database analysis
}

\author{
Dong Zeng ${ }^{1 \#}$, Xianghua Zeng ${ }^{2 \#}$, Jun Duan ${ }^{3}$, Diangang Chen ${ }^{1}$, Bo Zhu ${ }^{1}$ \\ ${ }^{1}$ Institute of Cancer, Xinqiao Hospital, Army Military Medical University, Chongqing, China; ${ }^{2}$ Department of Medical Oncology, Chongqing \\ University Cancer Hospital, Chongqing, China; ${ }^{3}$ Department of Emergency and Intensive Care Unit, The 991st Hospital of People's Liberation \\ Army of China, Xiangyang, China \\ Contributions: (I) Conception and design: B Zhu, D Zeng; (II) Administrative support: B Zhu; (III) Provision of study materials or patients: D Zeng; \\ (IV) Collection and assembly of data: D Zeng, X Zeng; (V) Data analysis and interpretation: D Zeng, X Zeng, D Chen; (VI) Manuscript writing: All \\ authors; (VII) Final approval of manuscript: All authors. \\ \#These authors contributed equally to this work. \\ Correspondence to: Bo Zhu. Institute of Cancer, Xinqiao Hospital, Army Military Medical University, Chongqing, China. Email: bo.zhu@tmmu.edu.cn.
}

Background: Primary hepatic angiosarcoma (PHA) is a rare malignant tumor. We explored the demographic features and prognostic factors of PHA.

Methods: We used the National Cancer Institute's Surveillance, Epidemiology, and End Results (SEER) database to extract patients diagnosed with PHA from 1975 to 2016. We used the Kaplan-Meier method and Cox proportional hazards regression to evaluate the risk factors for overall survival (OS) and disease-specific survival (DSS). The nomograms were constructed and validated using the concordance index (C-index) and calibration plots.

Results: In total, 366 patients were included in this study. The disease onset was hidden, and most patients already had advanced disease when diagnosed. The prognosis of PHA was very poor, and the overall 6-month, 1-year and 2-year survival rates were $20.3 \%, 12.8 \%$ and $9.3 \%$, respectively. Sex, age and surgery were all predictors of both OS and DSS in multivariate analysis. Women had better survival rates than men, and patients aged $<60$ years benefited from surgery in the multivariate models. The nomograms presented good accuracy, with C-index values of 0.679 and 0.665 for the OS and DSS prognostic models, respectively. The calibration plots showed good agreement between the nomogram predictions and actual observations.

Conclusions: PHA has a poor prognosis. Regular physical examinations are essential for the elderly. Patients aged $<60$ years could benefit from surgery. We constructed accurate nomograms to predict survival that can greatly benefit clinicians.

Keywords: Angiosarcoma; Surveillance, Epidemiology, and End Results program (SEER program); survival

Submitted Aug 23, 2020. Accepted for publication Nov 20, 2020.

doi: $10.21037 /$ tcr-20-2780

View this article at: http://dx.doi.org/10.21037/tcr-20-2780

\section{Introduction}

Angiosarcoma is a malignant tumor that originates from vascular endothelial cells. Approximately 5.4\% of cutaneous soft tissue sarcomas and $2 \%$ of soft tissue sarcomas are angiosarcomas. Angiosarcomas are widely distributed in the body, such as in the head and neck, breast, extremities, trunk, liver, heart, bone and spleen $(1,2)$. Primary hepatic angiosarcoma (PHA) is an extremely rare malignant neoplasm and accounts for only approximately $1 \%$ of primary malignant liver tumors (3). PHA has no specific etiologic agent in most patients, while exposure to vinyl chloride and ionizing radiation may be important risk factors $(4,5)$. Clinically, PHA shows various symptoms, such as abdominal pain, anorexia, fatigue, weight loss, fever, low back pain, jaundice, hemoperitoneum and acute 


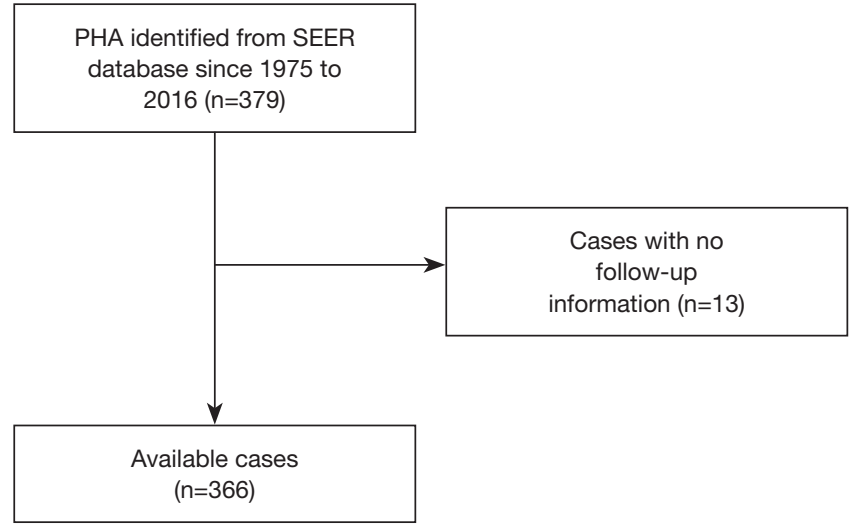

Figure 1 Schematic overview for patient identification. PHA, Primary hepatic angiosarcoma; SEER, Surveillance, Epidemiology, and End Results.

hepatic failure, although these symptoms are nonspecific $(6,7)$. Often, no specific symptoms are observed, and the levels of tumor markers are usually not elevated in the early stages of the disease, such as alpha fetoprotein (AFP), carcinoembryonic antigen (CEA), cancer antigen 19-9 (CA19-9), and cancer antigen 125 (CA125), making PHA difficult to diagnose (8). Histopathological diagnosis is the gold standard for PHA. In hematoxylin and eosin staining, the neoplastic cells are represented as marked nuclear pleomorphism with a spindle shape. Endothelial markers such as CD31, CD34 and ERG are reliable immunohistochemical markers $(9,10)$. The nonspecific symptoms of PHA and rapid progression result in a poor prognosis (11). PHA has a median survival of no more than 6 months (12). Surgery is usually the preferred method of treatment, and surgery combined with chemotherapy or chemotherapy alone are other choices in the clinical setting (13-15). However, no effective treatment guidelines have been established because of the low incidence and aggressive nature of PHA.

Currently, most studies are case reports and case series due to the rarity of PHA and are seriously limited by small patient numbers. Demographic and clinical prognostic factors were rarely considered. The Surveillance, Epidemiology, and End Results (SEER) database can overcome the problem of insufficient sample size (16). It covers approximately $28 \%$ of the United States population. In this study, we analyzed the demographics and tumor characteristics of patients with PHA and the outcomes of overall and disease-specific survival (DSS); our findings will benefit clinicians and future studies. We present the following article in accordance with the STROBE reporting checklist (available at http://dx.doi. org/10.21037/tcr-20-2780).

\section{Methods}

\section{Data source}

We retrieved data from the latest version of the SEER 18 database released in August 2019 using SEER*Stat software (version 8.3.6). All patient information was deidentified and publicly available, so institutional review board approval was not needed. The study was conducted in accordance with the Declaration of Helsinki (as revised in 2013).

\section{Patient selection}

The inclusion criteria were as follows: patients with tumors located in the liver (site and morphology, TNM 7/CS v0204+ Schema $=$ liver); patients with angiosarcoma, as defined by the International Classification of Diseases for Oncology, 3rd edition (ICD-O-3) with the morphological code 9120; patients with specific prognostic data (Figure 1).

\section{Covariates}

First, demographic data, such as the age at diagnosis, sex, race and marital status, were collected. Next, we collected tumor characteristics, such as historic stage, CS tumor size, CS extension, CS lymph and CS metastasis. Finally, the treatment, survival months, status and cause of death were obtained. Age was categorized into $<50$, $50-59,60-69,70-79$ and $\geq 80$ years. Sex was classified as male or female. Year of diagnosis was coded as $<2,000$ or $\geq 2,000$ and further categorized into 1975-1979, 1980 1984, 1985-1989, 1990-1994, 1995-1999, 2000-2004, 2005-2009, 2010-2014 and 2014-2016. Marital status was categorized as married (including common law), single (never married), other (including separated, divorced and widowed) and unknown. We coded race as white, black and American Indian/AK Native, Asian/Pacific Islander. Because of the small sizes of the latter two groups, they were combined into the category of "other". The historic stage was classified as localized, regional, distant and unknown. Tumor sizes were coded as $<5 \mathrm{~cm}, \geq 5 \mathrm{~cm}$ and unknown. CS extension was categorized as T1, T2, T3, T4 and unknown. The AJCC stage was derived from the AJCC TNM $6^{\text {th }}$ edition and defined as $1,2,3$ or 4 . DSS was defined as the time from diagnosis to death caused by 


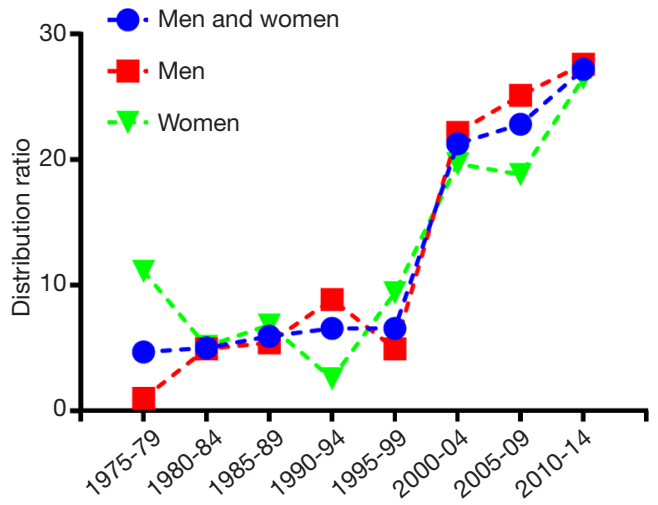

Figure 2 Analysis distribution of primary hepatic angiosarcoma patients from 1975 to 2014.

PHA. Overall survival (OS) was defined as the time from diagnosis to death from any cause.

\section{Statistical analysis}

The baseline characteristics were compared using chisquared test. Kaplan-Meier curves were constructed to estimate the OS and DSS, and log-rank tests were used to determine the significance of any differences. Univariate and multivariate Cox proportional hazards analyses were used to determine the independent prognostic predictors for OS and DSS, and the hazard ratios and $95 \%$ confidence intervals were shown. We used SPSS ver. 20.0 (IBM Corporation) and GraphPad Prism ver. 7.02 to conduct statistical analysis. A P value $<0.05$ was considered statistically significant.

A nomogram for the final prognostic factors associated with the 6-month and 1-year survival rates was established. The predictive performance was evaluated using the concordance index (C-index) and calibration plots to compare the nomogram predictions with the observed outcomes. R software ver.3.6.1 was used to construct the nomograms.

\section{Results}

\section{Demographic and clinicopathological characteristics of PHA}

As shown in Figure 2, we computed the number of PHA patients in 5-year intervals (1975-1979, 1980-1984, 19851989, 1990-1994, 1995-1999, 2000-2004, 2005-2009, 2010-2014) and found increasing trends in the number of both men and women.

As shown in Table 1, 366 patients were included in this study, 235 men and 131 women, at a ratio of 1.79:1. The patients were mainly middle-aged and elderly at the time of diagnosis, and the average age of PHA patients was 62.2 years. Additionally, $79.2 \%$ of the patients were older than 50 years and $13.7 \%$ were older than 80 years. The race distribution was white $(79.0 \%)$, black (4.6\%) and other $(16.4 \%)$, indicating that this study mainly reflected the characteristics of white Americans. Most patients were married $(58.2 \%)$, and $16.7 \%$ of the subjects were single. Most of the patients were diagnosed after the year 2000, but this finding does not indicate an increase in the incidence. Tumor sizes were categorized into $<5 \mathrm{~cm}, \geq 5$ $\mathrm{cm}$ and unknown, and the proportions were $6.6 \%, 26.0 \%$ and $67.5 \%$, respectively. Regarding to tumor extent, most of the patients were in stage T2, although some were missing these data. Most of the patients did not have lymph node metastasis. Among those with AJCC stages recorded, $10.1 \%$ had stage I, $19.9 \%$ had stage II, $2.5 \%$ had stage III and $16.9 \%$ had stage IV disease. Regarding the historic stage, $24.6 \%$ of the patients had local stage disease, $26.0 \%$ had regional stage disease, and $111(30.3 \%)$ patients had distant stage disease. Regarding treatment, nearly six times as many patients $(64.8 \%) \mathrm{did}$ not receive surgery as those who received surgery (11.2\%). We compared the patients' baseline characteristics between those who died because of the tumor and those who survived or died because of unrelated causes, and differences were found in year of diagnosis, tumor size, distant metastasis and surgery.

\section{Survival}

The prognosis of PHA was very poor (Table 2), with a median survival of only 1 month. The overall 6-month, 1-year and 2-year survival rates were 20.3\% (95\% CI: $16.2-24.4 \%), 12.8 \%$ (95\% CI: $9.3-16.3 \%)$ and $9.3 \%(95 \%$ CI: 6.2-12.4\%), respectively. The disease-specific 6-month, 1-year and 2-year survival rates were $13.6 \%$ (95\% CI: 9.5-17.7\%), 6.4\% (95\% CI: 3.5-9.6\%) and 3.4\% (95\% CI: $1.2-5.6 \%)$, respectively.

In univariate analysis, sex, age and surgery were all predictors of both OS and DSS (Table 3 and Figure 3). Male patients had a worse prognosis than female patients. In general, the younger patients had better survival than the older patients. Survival was significantly better for patients who had undergone surgery than for those who did not. 
Table 1 Comparison of the baseline patient characteristics between patients who died because of the tumor and those who survived or died because of unrelated causes

\begin{tabular}{|c|c|c|c|c|}
\hline Characteristic & Total & Tumor-related death & Alive or unrelated cause of death & $P$ value \\
\hline Male & $235(64.2 \%)$ & 175 & 60 & \\
\hline Female & $131(35.8 \%)$ & 90 & 41 & \\
\hline Age, years & & & & 0.396 \\
\hline $50-59$ & $69(18.9 \%)$ & 48 & 21 & \\
\hline $60-69$ & $88(24.0 \%)$ & 69 & 19 & \\
\hline $70-79$ & $85(23.2 \%)$ & 56 & 29 & \\
\hline$>80$ & $50(13.7 \%)$ & 36 & 14 & \\
\hline Black & $17(4.6 \%)$ & 13 & 4 & \\
\hline Other & $60(16.4 \%)$ & 46 & 14 & \\
\hline Marital status & & & & 0.577 \\
\hline Marred & $213(58.2 \%)$ & 157 & 56 & \\
\hline Single & $61(16.7 \%)$ & 41 & 20 & \\
\hline Other & $79(21.6 \%)$ & 59 & 20 & \\
\hline NA & $13(3.6 \%)$ & 8 & 5 & \\
\hline Year of diagnosis & & & & 0.011 \\
\hline NA & $247(67.5 \%)$ & 189 & 58 & \\
\hline Tumor extent & & & & 0.060 \\
\hline $\mathrm{T} 1$ & 47 (12.8\%) & 29 & 18 & \\
\hline T2 & 107 (29.2) & 74 & 33 & \\
\hline T3 & $1(0.3 \%)$ & 0 & 1 & \\
\hline $\mathrm{T} 4$ & $11(3.0 \%)$ & 10 & 1 & \\
\hline NA & $200(54.6 \%)$ & 152 & 48 & \\
\hline LN metastasis & & & & 0.100 \\
\hline No & $193(52.7 \%)$ & 131 & 62 & \\
\hline Yes & $8(2.2 \%)$ & 7 & 1 & \\
\hline
\end{tabular}

Table 1 (continued) 
Table 1 (continued)

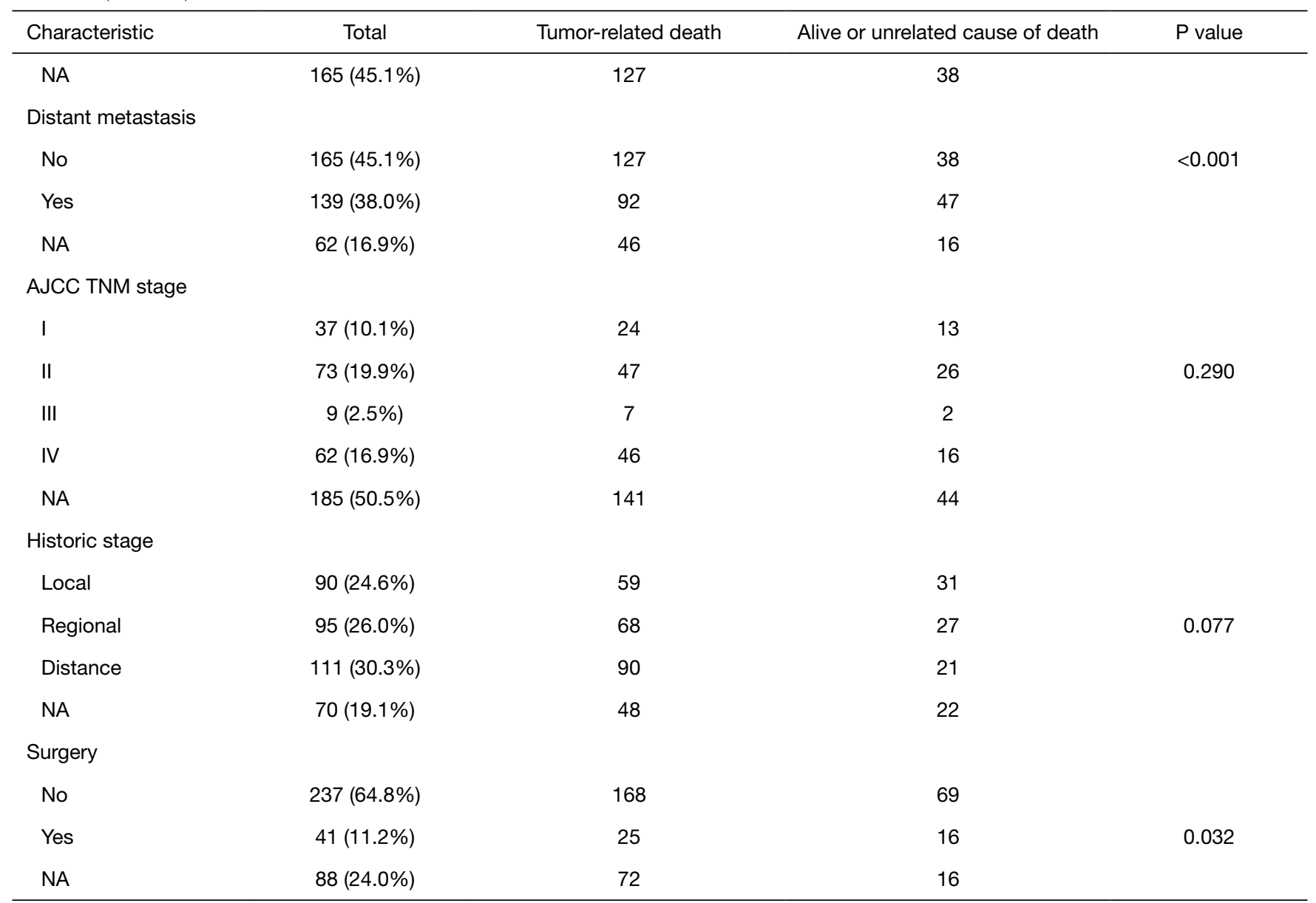

$\mathrm{Na}$, not available; LN metastasis, lymph node metastasis.

Table 2 Overall survival and disease-specific survival as estimated by Kaplan-Meier analysis

\begin{tabular}{lccc}
\hline Variables & 6-month, \% (95\% Cl) & 1-year, \% (95\% Cl) & 2-year, \% (95\% Cl) \\
\hline Overall survival (OS) & $20.3(16.2-24.4)$ & $12.8(9.3-16.3)$ & $9.3(6.2-12.4)$ \\
Disease-specific survival (DSS) & $13.6(9.5-17.7)$ & $6.4(3.5-9.6)$ & $3.4(1.2-5.6)$ \\
\hline
\end{tabular}

Tumor size and historic stage were predictors of OS but not DSS. However, tumor extent, LN metastasis, distant metastasis and TNM stage did not significantly influence survival.

To further study the prognostic factors for PHA, we constructed multivariate models for OS and DSS. Women had better OS than men $(\mathrm{P}=0.018)$, and sex had a significant effect on DSS ( $\mathrm{P}=0.043$; Table 4). Multivariate analysis revealed that age was an important factor affecting survival. We divided the patients into two age groups: $<60$ years and $\geq 60$ years; we then studied their baseline characteristics. The basic characteristics of the two groups were similar, and no significant difference was found in the composition (Table 5). Overall, older patients had a worse prognosis than younger patients. Tumor size was a predictor of OS but not DSS. Patients with a distant historic stage had worse OS than those with a local historic stage. Patients who received surgery had a much better prognosis than those who did not. However, TNM stage was not significantly correlated with prognosis. 
Table 3 Univariate analyses of overall survival and disease-specific survival in PHA patients

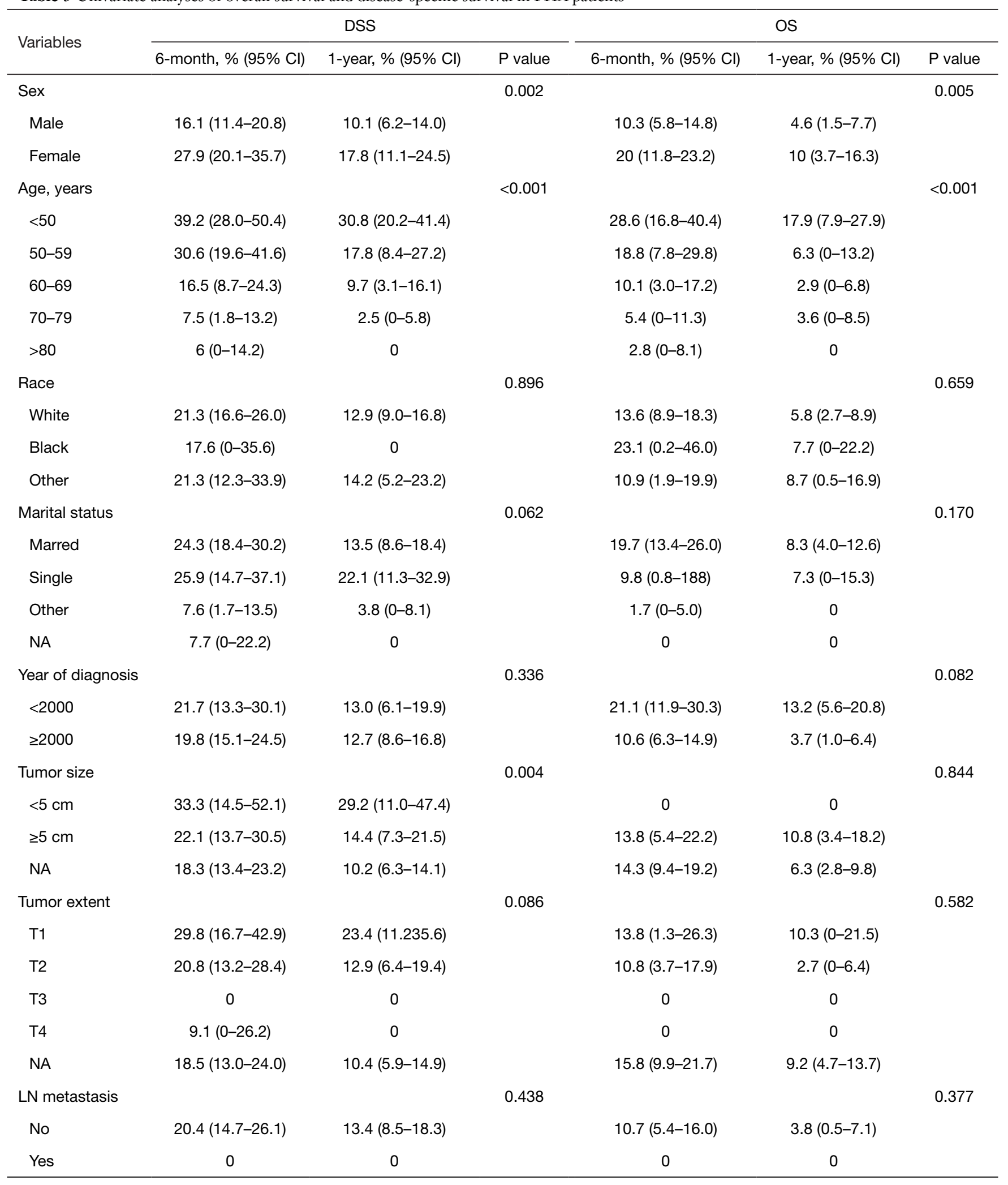

Table 3 (continued) 
Table 3 (continued)

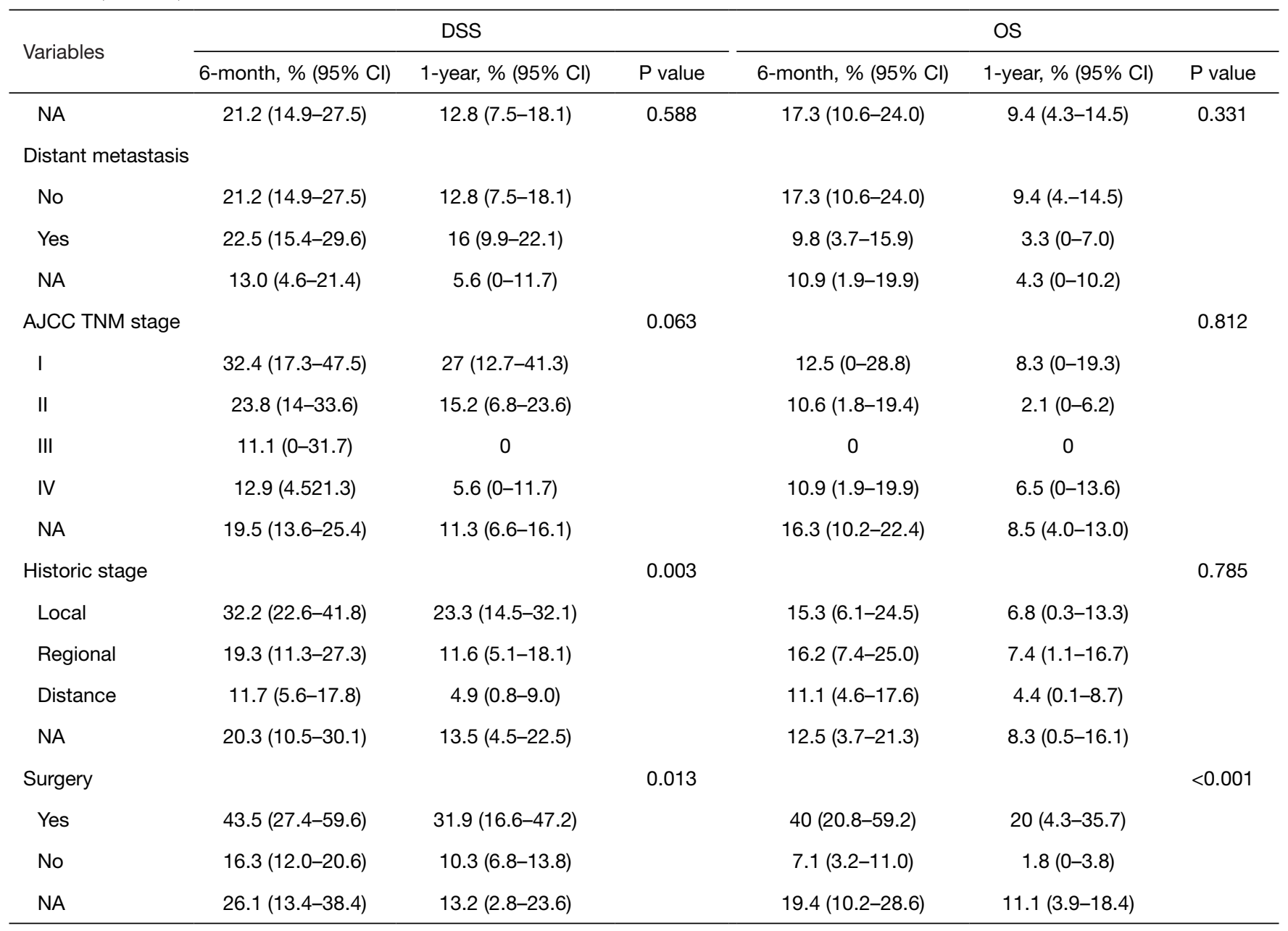

NA, not available; LN metastasis, lymph node metastasis.

To identify the ages at which patients can benefit from surgery, we conducted an exploratory subgroup analysis. Patients aged $<60$ years benefited more from surgery in terms of OS and DSS than those aged $\geq 60$ years (Table 6).

Furthermore, nomograms were established to predict the 0.5 - and 1-year OS and DSS (Figure 4). In the OS prognostic model, the nomograms presented good accuracy, with a C-index value of 0.679 (95\% CI: $0.642-0.716)$, and the C-index value was 0.665 (95\% CI: $0.618-0.712$ ) in the DSS prognostic model. The calibration plots for the 0.5 and 1-year OS and 0.5- and 1-year DSS demonstrated fair agreement between the actual observations and nomogrampredicted probabilities (Figure 5).

\section{Discussion}

PHAs are rare malignant neoplasms, and few studies have been published on PHA. Most of the studies are case reports, and few researchers have analyzed the clinical features and long-term follow-up reports of this disease in detail. We conducted a population-based study to identify the clinical characteristics and investigate the factors related to the prognosis of PHA. To our best of knowledge, this study has the largest number of patients currently, and the findings will benefit doctors and future researchers.

Some studies have reported that PHA accounts for nearly $2 \%$ of primary liver tumors (17). However, we 

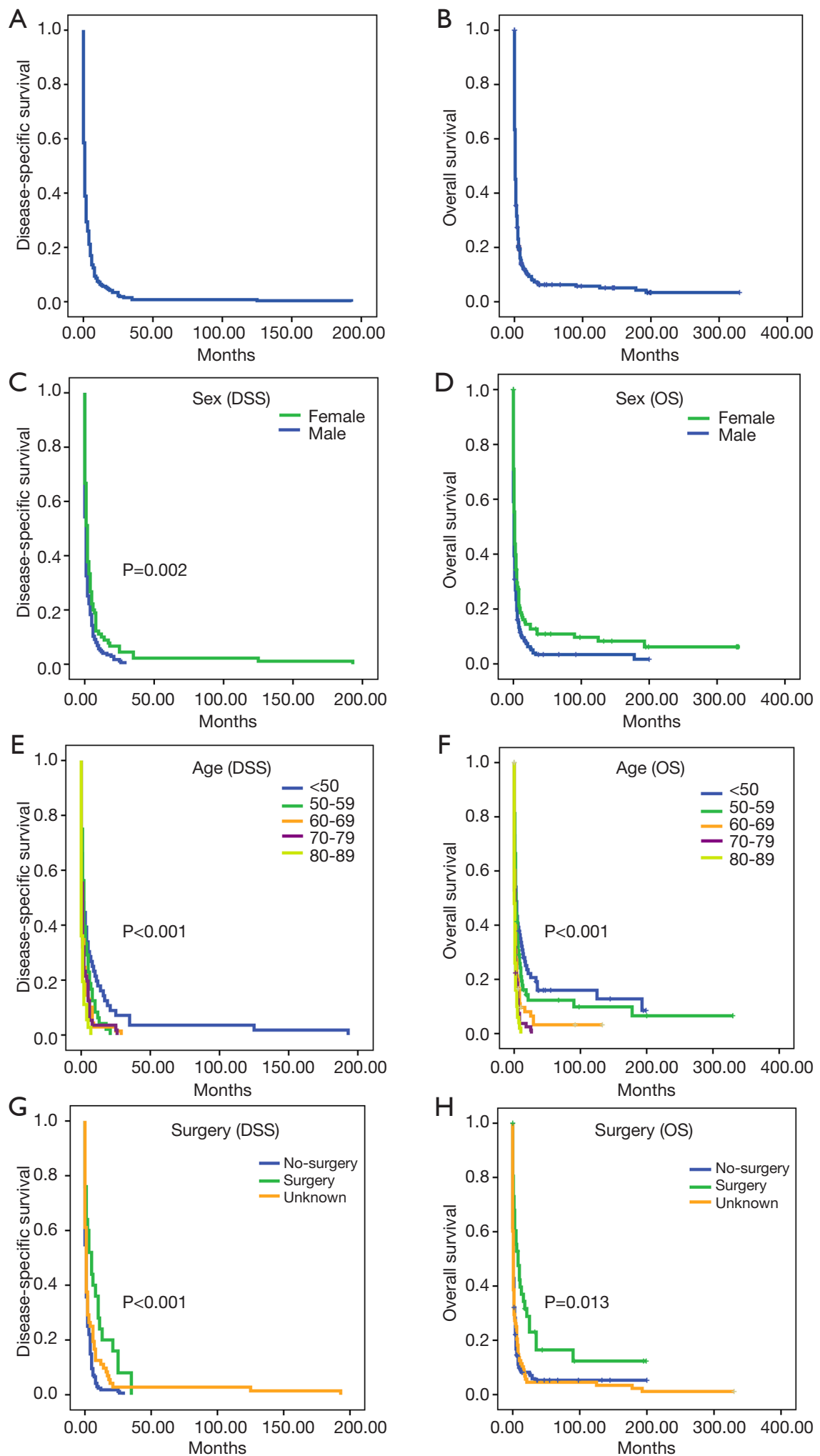

Figure 3 Kaplan-Meier survival plots for primary hepatic angiosarcoma. (A) Overall survival (OS). (B) Disease-specific survival (DSS). (C,D) Sex is a predictor of both OS and DSS. (E,F). Age is a predictor of both OS and DSS. $(\mathrm{G}, \mathrm{H})$ Surgery is a predictor of both OS and DSS. 
Table 4 Multivariate analyses of overall survival and disease-specific survival in PHA

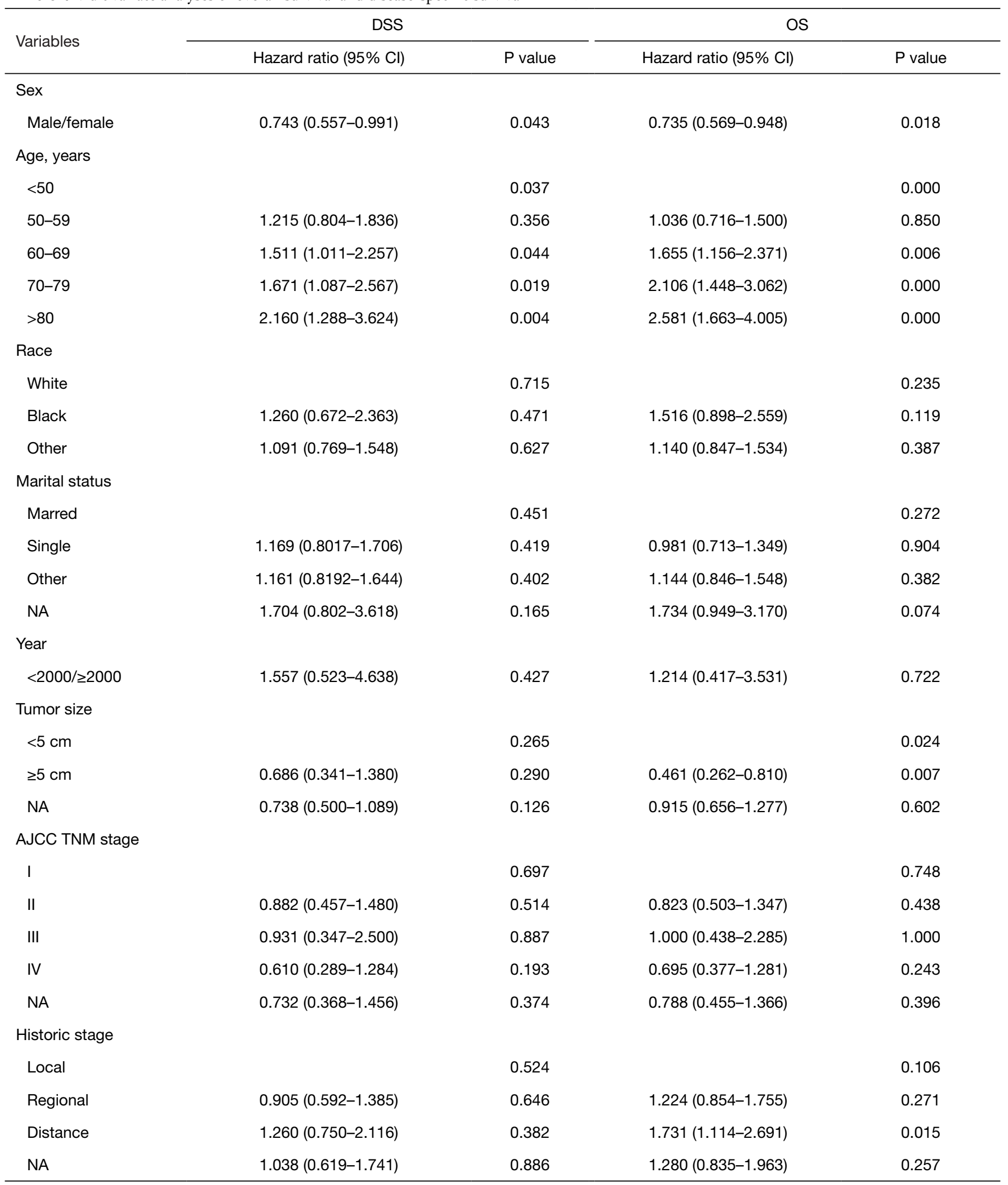

Table 4 (continued) 
Table 4 (continued)

\begin{tabular}{|c|c|c|c|c|}
\hline Variables & \multicolumn{2}{|l|}{ DSS } & \multicolumn{2}{|c|}{ Os } \\
\hline \multicolumn{5}{|l|}{ Surgery } \\
\hline No & & 0.047 & & 0.016 \\
\hline Yes & $0.571(0.348-0.938)$ & 0.027 & $0.586(0.392-0.877)$ & 0.009 \\
\hline
\end{tabular}

DSS, disease-specific survival; OS, overall survival; NA, not available.

identified more than 120,000 liver cancer patients in the SEER database, and fewer than 400 had PHA, accounting for less than $0.3 \%$. This evidence supports the idea that PHA is rare. Our study showed that the number of patients increased significantly after the start of the 21 st century, likely because of the direct link to improved diagnostic methods, but the reasons for this increase must be further studied. According to a previous systematic review of our team, the results showed a slight predominance of men, with a sex ratio of 1.88 , a finding that was similar to this study. Additionally, $12.8 \%$ of patients had a history of vinyl chloride exposure (18). Therefore, we speculate that occupational exposure to vinyl chloride or other carcinogens may be the cause of the difference in prevalence between men and women. Unfortunately, we did not know the exact occupational exposure rates between men and women in the present study.

The average age of PHA patients was 62.2 years. Wilson GC reported that the median age was 63.7 years (19), a finding that was similar to ours. Therefore, both results indicated that PHA mainly occurs in the elderly population.

PHA lacks specific clinical symptoms and signs in the early stage, leading to delays in diagnosis and treatment. Lesions are not identified in some patients until the tumor ruptures (20-22). Among the 119 patients with clear records of tumor size, the number of patients with tumor volumes $\geq 5 \mathrm{~cm}$ was more than three times that of patients with tumor volumes $<5 \mathrm{~cm}$ in our study. Although only a few patients had lymph node metastasis, nearly $40 \%$ of the patients had distant tumor metastasis, demonstrating the highly invasive nature of PHA. Regarding the historic stage, more than one-third of the patients were in the distant stage, also supporting the findings of the poor prognosis of PHA. Thus, the onset of the disease is insidious and the elderly should have regular physical examinations.
The delayed diagnosis and highly malignant characteristics of the tumor result in a very poor prognosis. Some studies have reported a median survival of approximately 6 months, and only $3 \%$ of patients survived for more than 2 years (23). According to our study, the median OS was only 1 month, with $12.8 \%$ of the patients surviving for more than 1 year. Moreover, the 1 -year DSS rate was only $6.4 \%$, which was an alarming result. This finding further demonstrates that the prognosis is very poor, and doctors should lower the expectations of patients or their families. Currently, only a few systematic studies have investigated the prognostic factors for this disease. Li et al. found that a small tumor size $(<10 \mathrm{~cm})$ was the only significant favorable factor for OS, while sex, age, hepatectomy, tumor rupture and adjuvant chemotherapy had no significant influence on survival (24). These results were obtained with univariate analysis. In our study, age, sex and surgery were all prognostic predictors in univariate analysis. The patients who were younger, female or received surgery had better survival than their counterparts. Tumor size and historic stage were prognostic predictors for DSS. However, tumor extent, LN metastasis, distant metastasis and TNM stage did not significantly influence survival in univariate analysis. Considering the limitations of univariate Cox analysis, we applied multivariable Cox analysis to further study the relevant factors. Consistent with univariate Cox analysis, age, sex and surgery were significant prognostic factors. Tumor size and distant historic stage were predictors of OS but not DSS. TNM stage had no significant influence on prognosis. According to a recent SEER study, age, gender, marital status, primary site, tumor size, historic stage and surgery were predictors of OS in angiosarcoma (25). The reason for the difference in results may be that the characteristics of angiosarcoma at different locations may be inconsistent.

Currently, several treatments for this disease, such as surgery, adjuvant chemotherapy, liver transplantation, 
Table 5 Comparison of the baseline patient characteristics between patients aged $<60$ and $\geq 60$ years

\begin{tabular}{|c|c|c|c|c|c|c|}
\hline Variables & \multicolumn{3}{|c|}{ OS } & \multicolumn{3}{|c|}{ DSS } \\
\hline Sex & & & 0.058 & & & 0.354 \\
\hline Male & 83 & 152 & & 65 & 110 & \\
\hline Female & 60 & 71 & & 39 & 51 & \\
\hline White & 111 & 178 & & 79 & 127 & \\
\hline Black & 10 & 7 & & 9 & 4 & \\
\hline Other & 22 & 38 & & 16 & 30 & \\
\hline Year of diagnosis & & & 0.317 & & & 0.249 \\
\hline Size & & & 0.943 & & & 0.284 \\
\hline$<5 \mathrm{~cm}$ & 36 & 59 & & 24 & 41 & \\
\hline$\geq 5 \mathrm{~cm}$ & 9 & 15 & & 2 & 9 & \\
\hline NA & 98 & 149 & & 78 & 111 & \\
\hline AJCC TNM stage & & & 0.389 & & & 0.085 \\
\hline I & 16 & 21 & & 9 & 15 & \\
\hline II & 23 & 50 & & 12 & 35 & \\
\hline III & 2 & 7 & & 1 & 6 & \\
\hline Distance & 53 & 58 & & 45 & 45 & \\
\hline NA & 23 & 47 & & 15 & 33 & \\
\hline Surgery & & & 0.107 & & & 0.287 \\
\hline No & 84 & 153 & & 60 & 108 & \\
\hline Yes & 21 & 20 & & 12 & 13 & \\
\hline NA & 38 & 50 & & 32 & 40 & \\
\hline
\end{tabular}

DSS, disease-specific survival; OS, overall survival; NA, not available.

transcatheter arterial embolization, transcatheter arterial chemoembolization and symptomatic supportive treatment are available, but no consensus exists on standard treatment (26-31). To date, the mainstay of treatment comprises radical tumor resection or hepatic resection when the patient's general physical condition is adequate (32). In our study, we found that patients who received surgery had much better survival than those who did not, further 
Table 6 Multivariate analyses of DSS and OS stratified by age

\begin{tabular}{|c|c|c|c|c|}
\hline Variables & \multicolumn{2}{|c|}{ DSS } & \multicolumn{2}{|c|}{ OS } \\
\hline \multicolumn{5}{|l|}{ Sex } \\
\hline Male/female & 0.003 & 0.584 & 0.000 & 0.479 \\
\hline \multicolumn{5}{|l|}{ Race } \\
\hline Black & 0.974 & 0.671 & 0.395 & 0.509 \\
\hline Other & 0.565 & 0.718 & 0.690 & 0.407 \\
\hline \multicolumn{5}{|l|}{ Marital status } \\
\hline Marred & 0.459 & 0.470 & 0.218 & 0.525 \\
\hline NA & 0.159 & 0.607 & 0.102 & 0.236 \\
\hline \multicolumn{5}{|l|}{ Year of diagnosis } \\
\hline$<2000 / \geq 2000$ & 0.214 & 0.680 & 0.483 & 0.567 \\
\hline \multicolumn{5}{|l|}{ Size } \\
\hline$<5 \mathrm{~cm}$ & 0.733 & 0.540 & 0.030 & 0.223 \\
\hline$\geq 5 \mathrm{~cm}$ & 0.683 & 0.513 & 0.019 & 0.083 \\
\hline NA & 0.453 & 0.282 & 0.702 & 0.461 \\
\hline \multicolumn{5}{|l|}{ AJCC TNM stage } \\
\hline NA & 0.286 & 0.408 & 0.214 & 0.158 \\
\hline \multicolumn{5}{|l|}{ Historic stage } \\
\hline Local & 0.758 & 0.914 & 0.604 & 0.896 \\
\hline Regional & 0.982 & 0.635 & 0.400 & 0.725 \\
\hline Distance & 0.429 & 0.888 & 0.612 & 0.492 \\
\hline NA & 0.928 & 0.893 & 0.782 & 0.519 \\
\hline \multicolumn{5}{|l|}{ Surgery } \\
\hline No & 0.003 & 0.619 & 0.049 & 0.199 \\
\hline Yes & 0.004 & 0.334 & 0.045 & 0.073 \\
\hline NA & 0.787 & 0.873 & 0.645 & 0.878 \\
\hline
\end{tabular}

DSS, disease-specific survival; OS, overall survival; NA, not available. 
A

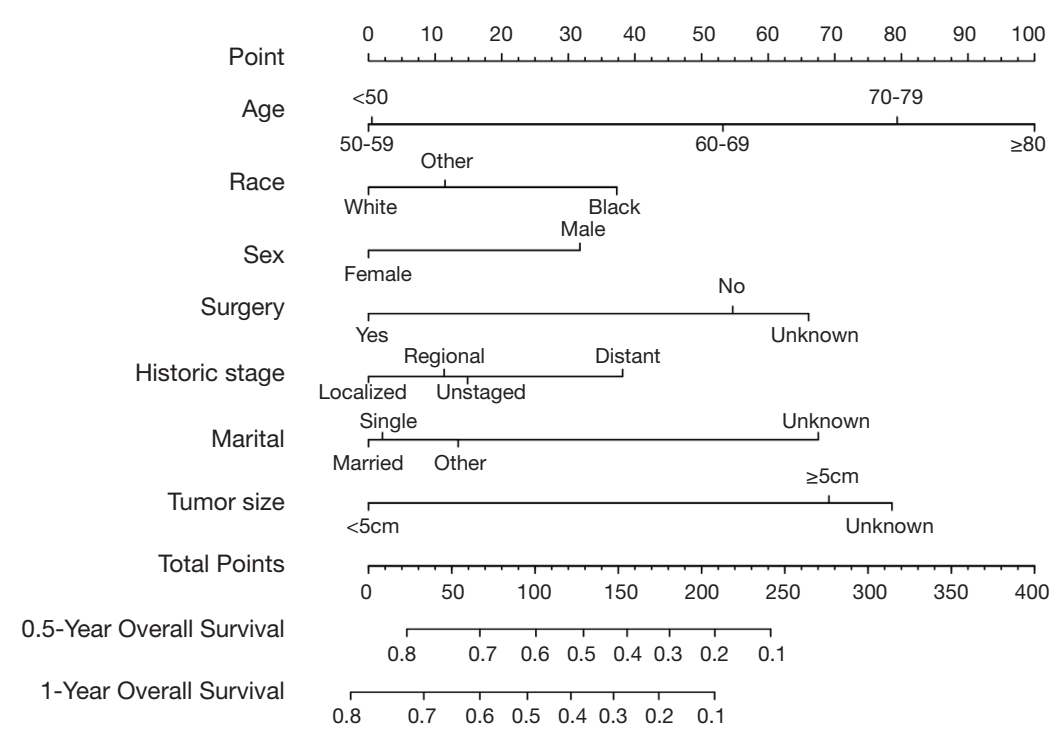

B

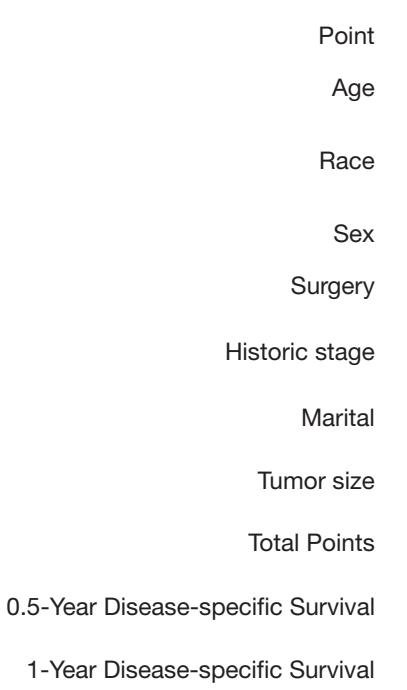

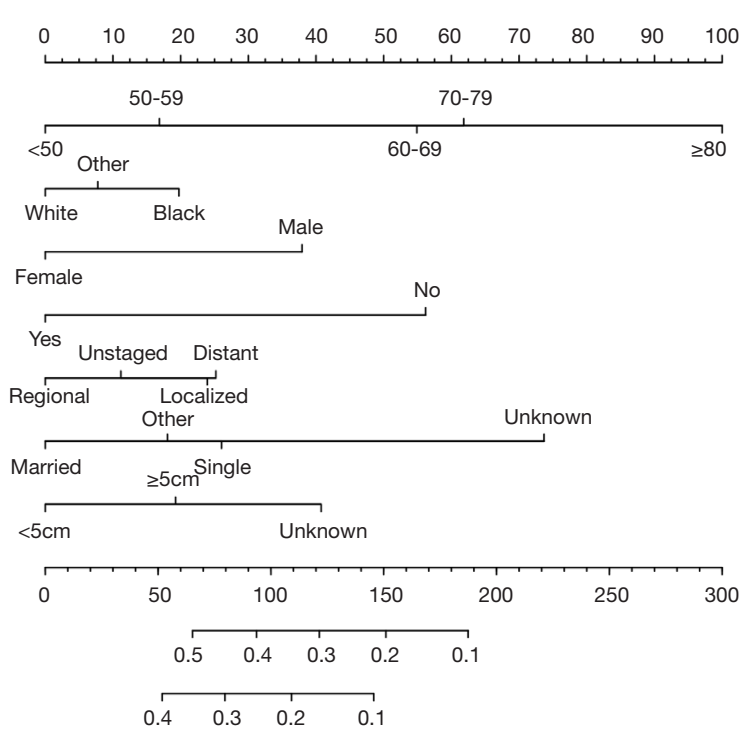

Figure 4 Nomograms for predicting patients' survival. (A) Nomograms predicting the 0.5 - and 1-year overall survival. (B) Nomograms predicting the 0.5 - and 1 -year disease-specific survival.

supporting the choice of surgery as the primary treatment. Moreover, according to exploratory subgroup analysis, patients aged $<60$ years benefit from surgery, providing a more specific reference for clinicians.

Nomograms have been used to assess OS and cancerspecific survival in cancer patients and serve as a reliable tool to help clinicians evaluate patient prognosis $(33,34)$. Patient scores are obtained based on the corresponding indicators, and the total scores can be used to assess the prognosis. To our best knowledge, no nomogram has been constructed to predict OS and DSS in PHA. In this study, we successfully constructed nomograms and evaluated them using $\mathrm{C}$-index values and calibration plots. The $\mathrm{C}$-index values ranged from 0.5 to 1.0 . A $\mathrm{C}$-index value of 0.5 indicates random chance, and 1.0 indicates perfect discriminative ability (35). The $\mathrm{C}$-index values were 0.679 (95\% CI: 0.642-0.716) and 0.665 (95\% CI: 0.618-0.712) for the OS and DSS nomograms, respectively, indicating 

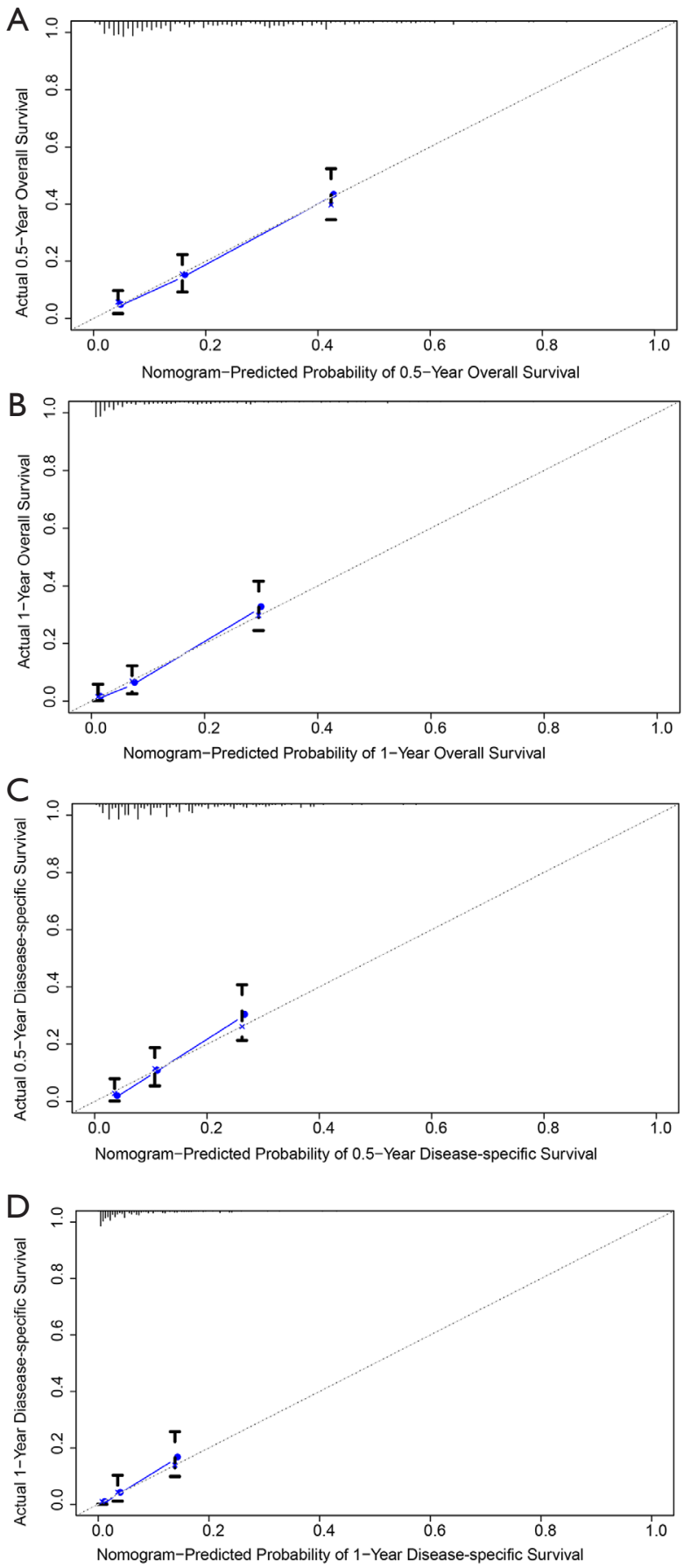

Figure 5 Calibration curves of the nomogram for predicting patients' survival. (A) Half-year overall survival (OS). (B) One-year OS. (C) Half-year disease-specific survival (DSS). (D) One-year DSS. relatively high accuracy. Additionally, the calibration plots showed satisfactory results. Clinicians can use this model to evaluate the prognosis of patients more accurately.

This study possessed several limitations. First, data were missing in the categories of tumor size, tumor extent, lymph node metastasis and historic stage, which may have caused information bias and decreased the accuracy of the nomograms. Second, the SEER data lacked information on clinical symptoms, which may reflect the severity of the disease. Third, the SEER data mainly represented white US individuals, affecting the generalization of our results to populations of different ethnicities. Additional studies with multi-institutional cohorts are needed in the future. Additionally, the study was retrospective, and prospective cohorts will be needed to validate the accuracy of the nomograms.

\section{Conclusions}

In this study, we used the SEER database to determine the clinicopathological characteristics, treatments and outcomes of the largest cohort of PHA patients to date. The prognosis of the disease is poor, and we suggest that the elderly should have regular physical examinations. We identified age and sex as important factors affecting survival. Additionally, patients aged $<60$ years benefited from surgery. Finally, we constructed accurate nomograms to predict survival, and these visual representations of the prognostic factors may greatly benefit clinicians.

\section{Acknowledgments}

Funding: None.

\section{Footnote}

Reporting Checklist: The authors have completed the STROBE reporting checklist Available at http://dx.doi. org/10.21037/tcr-20-2780

Conflicts of Interest: All authors have completed the ICMJE uniform disclosure form (available at http://dx.doi. org/10.21037/tcr-20-2780). The authors have no conflicts of interest to declare. 
Ethical Statement: The authors are accountable for all aspects of the work in ensuring that questions related to the accuracy or integrity of any part of the work are appropriately investigated and resolved. The study was conducted in accordance with the Declaration of Helsinki (as revised in 2013). All patient information was deidentified and publicly available, so institutional review board approval was not needed.

Open Access Statement: This is an Open Access article distributed in accordance with the Creative Commons Attribution-NonCommercial-NoDerivs 4.0 International License (CC BY-NC-ND 4.0), which permits the noncommercial replication and distribution of the article with the strict proviso that no changes or edits are made and the original work is properly cited (including links to both the formal publication through the relevant DOI and the license). See: https://creativecommons.org/licenses/by-nc-nd/4.0/.

\section{References}

1. Molina E, Hernandez A. Clinical manifestations of primary hepatic angiosarcoma. Dig Dis Sci 2003;48:677-82.

2. Young RJ, Brown NJ, Reed MW, et al. Angiosarcoma. Lancet Oncol 2010;11:983-91.

3. Wadhwa S, Kim TH, Lin L, et al. Hepatic angiosarcoma with clinical and histological features of Kasabach-Merritt syndrome. World J Gastroenterol 2017;23:2443-7.

4. Mark L, Dermore F, Creech JL Jr, et al. Clinical and morphologic features of hepatic angiosarcoma in vinyl chlorideworkers. Cancer 1976;37:149-63.

5. Goerlitz DS, Blancato J, Ramesh A, et al. Somatic mutation signatures in primary liver tumors of workers exposed to ionizing radiation. Sci Rep 2019;9:18199.

6. Singh G, Mills C, Asadi K, et al. Hepatic angiosarcoma as a cause of acute liver failure. BMJ Case Rep 2018;2018:bcr2018225896.

7. López R, Castro-Villabón D, Álvarez J, et al. Hepatic angiosarcoma presenting as acute liver failure in young adults. Report of two cases and review of literature. Case Reports in Clinical Medicine 2013;2:439-44.

8. Qiao Y, Yang J, Liu L, et al. Successful treatment with pazopanib plus PD-1 inhibitor and RAK cells for advanced primary hepatic angiosarcoma: a case report. BMC Cancer 2018;18:212.

9. Wang ZB, Yuan J, Chen W, et al. Transcription factor ERG is a specific and sensitive diagnostic marker for hepatic angiosarcoma. World J Gastroenterol
2014;20:3672-9.

10. Zhu YP, Chen YM, Matro E, et al. Primary hepatic angiosarcoma: A report of two cases and literature review. World J Gastroenterol 2015;21:6088-96.

11. Lin YH, Lin CC, Concejero AM, et al. Surgical experience of adult primary hepatic sarcomas. World J Surg Oncol 2015;13:87.

12. Zheng YW, Zhang XW, Zhang JL, et al. Primary hepatic angiosarcoma and potential treatment options. J Gastroenterol Hepatol 2014;29:906-11.

13. Kim HR, Rha SY, Cheon SH, et al. Clinical features and treatment outcomes of advanced stage primary hepatic angiosarcoma. Ann Oncol 2009;20:780-7.

14. Gunawardena SW, Trautwein LM, Finegold MJ, et al. Hepatic Angiosarcoma in a Child: Successful Therapy With Surgery and Adjuvant Chemotherapy. Med Pediatr Oncol 1997;28:139-43.

15. Ozden I, Bilge O, Erkan M, et al. Five years and 4 months of recurrence-free survival in hepatic angiosarcoma. J Hepatobiliary Pancreat Surg 2003;10:250-2.

16. Wang J, Li S, Liu Y, et al. Metastatic patterns and survival outcomes in patients with stage IV colon cancer: A population-based analysis. Cancer Med 2020;9:361-73.

17. Ito Z, Kajihara M, Kobayashi $Y$, et al. Hepatic Angiosarcoma Associated with Esophageal Variceal Hemorrhage. Case Rep Gastroenterol 2016;10:440-5.

18. Zeng D, Cheng J, Gong Z, et al. A pooled analysis of primary hepatic angiosarcoma. Jpn J Clin Oncol 2020;50:556-67.

19. Wilson GC, Lluis N, Nalesnik MA, et al. Hepatic Angiosarcoma: A Multi-institutional, International Experience with 44 Cases. Ann Surg Oncol 2019;26:576-82.

20. Lee SW, Song CY, Gi YH, et al. Hepatic angiosarcoma manifested as recurrent hemoperitoneum. World J Gastroenterol 2008;14:2935-8.

21. Ogawa M, Ae R, Sasahara T. Primary Hepatic Angiosarcoma: A Case Report with 10-Year Patient Medical Data. Case Rep Oncol 2017;10:851-6.

22. McLean CK, Squires JH, Reyes-Mugica M, et al. Hepatic Vascular Tumors in the Neonate: Angiosarcoma. J Pediatr 2018;193:245-248.e1.

23. Huang NC, Wann SR, Chang HT, et al. Arsenic, vinyl chloride, viral hepatitis, and hepatic angiosarcoma: A hospital-based study and review of literature in Taiwan. BMC Gastroenterol 2011;11:142.

24. Li DB, Si XY, Wan T, et al. A pooled analysis of treatment and prognosis of hepatic angiosarcoma in adults. Hepatobiliary Pancreat Dis Int 2018;17:198-203. 
25. Zhang C, Xu G, Liu Z, et al. Epidemiology, tumor characteristics and survival in patients with angiosarcoma in the United States: a population-based study of 4537 cases. Jpn J Clin Oncol 2019;49:1092-9.

26. Tsunematsu S, Muto S, Oi H, et al. Surgically Diagnosed Primary Hepatic Angiosarcoma. Intern Med 2018;57:687-91.

27. Terzi A, Deniz EE, Haberal N, et al. Hepatic angiosarcoma and liver transplant: a report of 2 cases with diagnosticdifficulties. Exp Clin Transplant 2014;12 Suppl 1:126-8.

28. Kamatani T, Iguchi H, Okada T, et al. Co-registered positron emission tomography/computed tomography and gadolinium-ethoxybenzyl-diethylenetriamine pentaacetic acid magnetic resonance imaging features of multiple angiosarcoma of the liver. Hepatol Res 2014;44:E297-303.

29. Maeda T, Tateishi U, Hasegawa T, et al. Primary hepatic angiosarcoma on coregistered FDG PET and CT images. AJR Am J Roentgenol 2007;188:1615-7.

30. Rowe K, Nehme F, Wallace J, et al. Primary Hepatic Angiosarcoma Mimicking Multifocal Liver Abscess

Cite this article as: Zeng D, Zeng X, Duan J, Chen D, Zhu B. Clinical characteristics of primary hepatic angiosarcoma outcomes: a SEER database analysis. Transl Cancer Res 2021;10(1):110-125. doi: 10.21037/tcr-20-2780 with Disseminated Intravascular Coagulation and Hemoperitoneum. Cureus 2017;9:e1293.

31. Fujii F, Kimura T, Tanaka N, et al. Hepatic Angiosarcoma with Kasabach-Merritt Phenomenon: A Case Report and Review of the Literature. Ann Hepatol 2018;17:655-60.

32. Lazăr DC, Avram MF, Romosan I, et al. Malignant hepatic vascular tumors in adults: Characteristics, diagnostic difficulties and current management. World J Clin Oncol 2019;10:110-35.

33. Zhang G, Li Z, Song D, et al. Nomograms to predict individual prognosis of patients with squamous cell carcinoma of the urinary bladder. BMC Cancer 2019;19:1200.

34. Zhang J, Yang Y, Fu X, et al. Development and validation of nomograms for prediction of overall survival and cancer-specific survival of patients of colorectal cancer. Jpn J Clin Oncol 2020;50:261-9.

35. Tang F, Zhang H, Lu Z, et al. Prognostic Factors and Nomograms to Predict Overall and Cancer-Specific Survival for Children with Wilms' Tumor. Dis Markers 2019;2019:1092769. 